\title{
Dental Referrals of Oncology Patients
}

Dr. Junima Rajkarnikar

Keywords: Dental referral; oncology; oral complications.

Head and neck cancer is the sixth most common cancer in the world. ${ }^{1}$ In South Asian countries like Nepal, it comprises nearly one fourth of all cases. ${ }^{2}$ As we know cancer patients can suffer from oral toxic effects of radiotherapy and chemotherapy, dental referral before the onset of these therapies is mandatory. While radiotherapy affects the cells within the irradiated area, chemotherapy affects cells even far away from the tumour site. ${ }^{3}$ Most common complications related to these therapies could be xerostomia, oral infections, fibrosis of the oral mucosa and osteoradionecrosis. Any abscesses should be treated and oral prophylaxis should be done especially in chronic periodontitis patients. ${ }^{4}$ During the immunosuppressive chemotherapy stage, periodontal infections which are otherwise self limiting, can be life threatening. Dental referrals before the antineoplastic therapy can improve the quality of life of the patient. Dental procedures should be completed at least two weeks prior to head/neck radiation. It is important to identify and treat sites of oral infections. ${ }^{3}$

Some of the most frequent side effects of chemotherapy are bone marrow suppression, resulting in leukopenia, thrombocytopenia and anaemia. Hyposialia of the cells of the salivary glands is mainly accountable for oral functional problems that can also lead to decrease in immunoglobulins A and G, eventually leading to development of mucositis. Osteonecrosis of the jaw (ONJ) is observed in patients with bisphosphonates. ONJ tends to appear more often after invasive dental manipulation like extractions so these should be carried out in advanced to avoid ONJ. ${ }^{3}$

The severity of the oral complications can be reduced significantly, if oral hygiene is stabilized beforehand. No elective dental treatment should be carried out during the active treatment phase. ${ }^{3}$ Dental fluoridation is recommended with use of $0.2 \%$ chlorhexidine mouthrinse after the antineoplastic therapy. Use of paracetamol is recommended, if required, during the active stage of the treatment as nonsteroidal antiinflammatory drugs could interact with immunosuppressors which can lead to gastric ulcers, nephrotoxicity or risk of bleeding. ${ }^{4}$ Even post the radiation therapy, the patient should be evaluated every six to eight weeks or as required up to first six months after the cancer therapy. Care should be taken to check the weakness in masticatory muscles in the irradiated areas. Mouth opening exercises should be encouraged. ${ }^{3}$

\section{Correspondence:}

Dr. Junima Rajkarnikar

Journal of Nepalese Society of Periodontology and Oral Implantology, Kathmandu, Nepal.

email:drjunima@gmail.com

\section{Citation}

Rajkarnikar J. Dental Referrals of Oncology Patients. J Nepal Soc Perio Oral Implantol. 2019;3(5):1.

\section{REFERENCES}

1. Warnakulasuriya S. Global epidemiology of oral and oropharyngeal cancer. Oral Oncol. 2009;45(4-5):309-16.

2. Gupta N, Gupta R, Acharya AK, Patthi B, Goud V, Reddy S, et al. Changing Trends in oral cancer - a global scenario. Nepal J Epidemiol. 2016;6(4);613-9.

3. Dental provider's Oncology Pocket guide. Prevention and management of oral complications. Available from: https://www.nidcr.nih. gov/sites/default/files/2017-09/oncology-guide-dental-provider_0.pdf

4. Chaveli López B, Gavaldá Esteve C, Sarrión Pérez MG. Dental treatment considerations in the chemotherapy patient. J Clin Exp Dent. 2011;3(1):e31-42. 